\title{
2D simulations of the line-driven instability in hot-star winds
}

\section{Approximations for the 2D radiation force}

\author{
L. Dessart ${ }^{1,3}$ and S. P. Owocki ${ }^{2}$ \\ 1 Max-Planck-Institut für Astrophysik, Karl-Schwarzschild-Strasse 1, 85748 Garching bei Munchen, Germany \\ e-mail: luc@as.arizona.edu \\ 2 Bartol Research Institute of the University of Delaware, Newark, DE 19716, USA \\ 3 Steward Observatory, University of Arizona, 933 North Cherry Avenue, Tucson, AZ 85721, USA
}

Received 27 January 2005 / Accepted 19 March 2005

\begin{abstract}
We present initial attempts to include the multi-dimensional nature of radiation transport in hydrodynamical simulations of the small-scale structure that arises from the line-driven instability in hot-star winds. Compared to previous 1D or 2D models that assume a purely radial radiation force, we seek additionally to treat the lateral momentum and transport of diffuse line-radiation, initially here within a 2D context. A key incentive is to study the damping effect of the associated diffuse line-drag on the dynamical properties of the flow, focusing particularly on whether this might prevent lateral break-up of shell structures at scales near the lateral Sobolev angle of ca. $1^{\circ}$. Based on 3D linear perturbation analyses that show a viscous diffusion character for the damping at these scales, we first explore nonlinear simulations that cast the lateral diffuse force in the simple, local form of a parallel viscosity. We find, however, that the resulting strong damping of lateral velocity fluctuations only further isolates azimuthal zones, leading again to azimuthal incoherence down to the grid scale. To account then for the further effect of lateral mixing of radiation associated with the radial driving, we next explore models in which the radial force is azimuthally smoothed over a chosen scale, and thereby show that this does indeed translate to a similar scale for the resulting density and velocity structure. Accounting for both the lateral line-drag and the lateral mixing in a more self-consistent way thus requires a multi-ray computation of the radiation transport. As a first attempt, we explore further a method first proposed by Owocki (1999), which uses a restricted 3-ray approach that combines a radial ray with two oblique rays set to have an impact parameter $p<R_{*}$ within the stellar core. From numerical simulations with various grid resolutions (and $p$ ), we find that, compared to equivalent 1-ray simulations, the high-resolution 3-ray models show systematically a much higher lateral coherence. This first success in obtaining a lateral coherence of wind structures in physically consistent $2 \mathrm{D}$ simulations of the radiative instability motivates future development of more general multi-ray methods that can account for transport along directions that do not intersect the stellar core.
\end{abstract}

Key words. hydrodynamics - line: formation - radiative transfer - stars: atmospheres - stars: early-type - stars: mass-loss

\section{Introduction}

The driving of hot-star winds by line-scattering of the star's radiation is understood to be highly unstable to small-scale radial perturbations (Lucy \& Solomon 1971; MacGregor et al. 1979; Owocki \& Rybicki 1984, 1985). This "Line-DrivenInstability" (LDI) has long been supposed to be a root cause of the extensive small-scale stochastic wind structure that has been inferred from various kinds of observational diagnostics (see reviews by, e.g., Owocki 1994; Feldmeier \& Owocki 1998; Dessart 2004a). For example, black troughs of saturated P-Cygni line profiles are understood to arise from the net backscattering of multiple line resonances occurring in a highly non-monotonic velocity field (Lucy 1982, 1984). Embedded wind shocks arising from such velocity variations are moreover thought to give rise to the soft X-ray emission often observed from such hot stars (Chlebowski 1989; Lucy \& White 1980;
Feldmeier et al. 1997). An even more direct diagnostic for such small-scale structure is the explicit, small-amplitude line profile variability often detected in high signal-to-noise $(S N$ 100-1000), optical spectra obtained by ground-based monitoring of recombination emission lines in both OB (Eversberg et al. 1998 on $\zeta$ Puppis) and WR stars (Robert 1992; Lépine \& Moffat 1999).

But a key limitation in developing quantitative tests for the association between such phenomena and the LDI is that, owing to the computational expense of evaluating non-local integrals needed for calculating the line-force, time-dependent dynamical simulations of the resulting nonlinear flow structure have for many years been limited to one dimension (1D) (Owocki et al. 1987; Feldmeier 1995; Feldmeier et al. 1997; Owocki \& Puls 1999; Runacres \& Owocki 2002). (See also Owocki 1999; and Gomez \& Williams 2003, for some previous 2D attempts.) These 1D simulations show development of 
extensive radial variations in both density and radial velocity, but are inherently incapable of determining the development of any corresponding variations in the lateral direction. In recent years, we have focused efforts on deriving empirical constraints on the multidimensional properties (particularly lateral scale) of wind structure (Dessart \& Owocki 2002a,b), and developing approaches for multidimensional simulation of the formation of such structure from the nonlinear evolution of the LDI (Dessart \& Owocki 2003, hereafter DO-03). The present effort builds on the "2D-H+1D-R" (denoting 2D Hydrodynamics, but with only 1D Radiation transport) approach of DO-03, now incorporating various approximate attempts to account for lateral transport and radiation forces associated with diffuse, scattered radiation, within similar 2D hydrodynamical simulation models.

A key aspect of line-driving in hot-star winds regards the Doppler shift associated with the wind acceleration and expansion. Along any given direction $\boldsymbol{n}$, this effectively desaturates the line-transfer on scales of the directional Sobolev length $l_{\boldsymbol{n}} \equiv v_{\text {th }} / \boldsymbol{n} \cdot \nabla(\boldsymbol{n} \cdot \boldsymbol{v})$, where $\boldsymbol{v}$ is the velocity, and $v_{\text {th }}$ is the ion thermal speed (typically a factor few smaller than the sound speed). In a smooth, supersonic wind with $v \gg v_{\text {th }}$, the Sobolev length $l_{n} \approx l_{0} \equiv r v_{\text {th }} / v$ is of order $v_{\text {th }} / v \ll 1$ smaller than the characteristic wind expansion scale $r$. The associated desaturation of the line-transfer then allows one to write a purely local expression for the line-acceleration $\boldsymbol{g}$ in terms of the local projected velocity gradient, $\boldsymbol{n} \cdot \nabla(\boldsymbol{n} \cdot \boldsymbol{v})$, averaged over directions to the source radiation from the stellar core ${ }^{1}$.

This "Sobolev approximation" (Sobolev 1960) provides the basic framework for the standard Castor et al. (1975, CAK) model for steady, spherically symmetric, line-driven stellar winds. Indeed, within this generalized, vector formulation, the purely local nature of the line-force makes even 3D timedependent simulations computationally feasible. As such, this vector Sobolev approach has been applied in numerous multidimensional models aimed at studying large-scale variations in line-driven winds. Associated with a rotational modulation of photospheric properties (Cranmer \& Owocki 1995; Dessart 2004b), they reproduced some of the key features of large scale variations observed in blueshifted absorption troughs of UV P-Cygni profiles in O-star (Howarth \& Prinja 1989) and Be-star (Grady et al. 1987) winds. Wind distortion due to stellar rotation (Owocki et al. 1996; Petrenz \& Puls 2000) also provides a key explanation for the observed polar-enhanced mass loss of line-driven winds such as those of the present day $\eta$ Car (Smith et al. 2003). The radiative-braking phenomenon (Gayley et al. 1997) advocates the potential of radiation to accelerate as well as decelerate stellar winds in massive binaries, a duality that proves essential to explain the geometry of wind-wind collisions in massive binary systems (van der Hucht \& Williams 1995).

\footnotetext{
${ }^{1}$ A further key simplification of this Sobolev approach is that the force associated with the diffuse, scattered component of radiation vanishes due to fore-aft symmetries of the Sobolev escape probability. As discussed below, the breaking of such symmetries for structures near the Sobolev scale leads to the diffuse "line-drag" effect first described by Lucy (1984).
}

Unfortunately, such a local Sobolev approach cannot be used to model structure arising from the LDI, since this occurs at scales near and below the Sobolev length (Owocki \& Rybicki 1984, 1985). Instead the line-force must be computed from a non-local radiation transport that can be cast approximately in terms of integral escape probabilities (Owocki \& Puls 1996). In $1 \mathrm{D}$ simulations, these escape probabilities can be evaluated from spatial integrals carried out along a restricted set of near radial directions intersecting the stellar core, repeated for a set of $n_{x} \approx 3 v_{\infty} / v_{\text {th }} \approx 1000$ frequencies that resolve the line thermal width over the full range of velocity shifts within the wind. This makes even 1D simulations of the LDI quite computationally expensive.

In $2 \mathrm{D}$ or $3 \mathrm{D}$, a proper treatment of the lateral transport requires such integration along a more complete set of oblique rays ranging from transverse to radial in direction. A severe complication is then that nonradial integrations from any given grid node do not generally intersect any other grid nodes. As such a straightforward "long characteristic" approach would require repeating the integration anew for each grid node, with a complex interpolation for the variation of the flow variables along the ray. For even a 2D grid of $n_{r}$ radial and $n_{\phi}$ azimuthal zones, this requires $n_{r} n_{\phi}$ integrals involving of order $n_{r} n_{x}$ operations for each of the set of $n_{\text {ray }}$ directions, giving an overall scaling of $n_{\text {ray }} n_{x} n_{r}^{2} n_{\phi}$ operations. For a typical case with $n_{\text {ray }} \approx 10, n_{\phi} \approx 100$, and $n_{x} \approx n_{r} \approx 1000$, this implies of order $10^{12}$ operations to evaluate the radiative force at each time step of a simulation model!

Such timing might be reduced somewhat by a "short characteristic" approach that builds up the local escape probabilities based on the evaluation in neighboring zones (e.g., van Noort et al. 2002). But before attempting to develop such a complex and costly general method, we explore here some more tractable, approximate treatments for the lateral radiation transport and associated force, aimed at gaining some initial insights into the key dynamical effects within 2D models.

In particular, a key issue in such instability simulations is taking proper account of the "line-drag" effect of the diffuse, scattered radiation (Lucy 1984; Owocki \& Rybicki 1985). In 1D simulations, the associated reduction in the net growth rate of the strong radial instability can be modeled via a "Smooth Source Function" (SSF; Owocki 1991; Owocki \& Puls 1996) method. This ignores any variations in the scattering source function, but accounts, through the radial integrations for the non-local escape probability, for small-scale, fore-aft asymmetries that give rise to this diffuse drag (Owocki \& Rybicki 1985). This nearly stabilizes the flow near the wind base, but in the outer wind there is still a strong net radial instability from driving by the direct radiation from the stellar core.

For lateral directions not intersecting the stellar core, the 3D linear stability analysis by Rybicki et al. (1990, hereafter ROC) shows that this diffuse line-drag leads to a strong net damping of velocity variations at scales near and below the lateral Sobolev length $l_{0}=r v_{\text {th }} / v$. In DO-03 we speculated that such damping might inhibit the lateral overturning of RayleighTaylor and thin-shell instabilities that break up radial shell structures, and so might lead to an overall lateral coherence at an associated angular scale $\Delta \phi_{0} \approx l_{0} / r=v_{\text {th }} / v \approx 0.01 \mathrm{rad} \approx 1^{\circ}$. 
This "Sobolev angle" is somewhat smaller than, but comparable to, the typical angle scale $\sim 3^{\circ}$ inferred for wind structure from analysis of line-profile variations in emission lines from Wolf-Rayet stars (Dessart \& Owocki 2002a,b).

To explore this and other effects that might influence the lateral scale of structure, the present paper carries out 2D instability simulations that account approximately for lateral transfer effects, including the diffuse line drag. Our initial approach (Sect. 2) uses a simple, local parallel viscosity formulation for the azimuthal diffuse line-force; as shown in Appendix A, the 3D linear stability analysis by ROC implies such a viscous scaling for the line force arising from azimuthal velocity perturbations on a scale near and above the lateral Sobolev length $l_{0}$. Results from our nonlinear simulations show that this lateral viscosity can indeed strongly damp azimuthal velocity variations, but does not, by itself, lead to a lateral coherence above the azimuthal grid scale.

Arguing then that such coherence might instead arise from lateral mixing of radiation associated with the radial driving, we next explore (Sect. 3) models with an azimuthal smoothing of the radial line-force, showing that this does lead to a comparable lateral smoothing of the resulting flow structure. To account more consistently for both effects, we finally examine fully non-local formulations of both the radial and azimuthal force obtained using a restricted, grid-aligned, "3-ray SSF method" first introduced by Owocki (1999). Initial results do show an extended lateral coherence of instability-generated structure, but inherent limitations in the ray coverage and outer radial grid resolution in the method leave uncertain the broad applicability of these 3-ray simulations. We conclude (Sect. 5) with a brief summary of results and their implications for future development of generalized multi-ray methods to account for multi-dimensional transport in simulations of structure arising from the LDI.

\section{Radiative viscosity model for the lateral, diffuse force}

As first derived in the 3D linear stability analysis by ROC, a key multidimensional effect of the diffuse radiation is the tendency to strongly damp lateral velocity perturbations. Appendix A shows that this can be cast in a general analytic form (Eq. (A.3)) that reduces to a simple viscous diffusion (Eq. (A.5)) for variations on scales near or above the (quite small) Sobolev length, $l_{0} \approx r v_{\text {th }} / v \approx 0.01 r$. To now account for the dynamical effects of diffuse lateral transport in nonlinear simulations of wind structure, let us here mimic this scaling from the linear perturbation analysis, and so assume that the azimuthal component of the diffuse line-force can be approximated as a standard parallel viscosity term,

$g_{\mathrm{diff}, \phi}(\boldsymbol{r})=\alpha_{\mathrm{vis}}\left(v_{\mathrm{th}} r\right) \frac{\partial^{2} v_{\phi}(\boldsymbol{r})}{r^{2} \partial \phi^{2}}$.

Here $\alpha_{\text {vis }}$ is a dimensionless parameter to control the overall strength of the viscous dissipation in terms of the assumed dimensional scale $v_{\mathrm{th}} r$ for the kinematic viscosity. This is indeed the scaling derived from the linear perturbation analysis (cf. Eq. (A.5)), with $\alpha_{\text {vis }} \approx s \alpha / 6$ a fraction of order a few tenths.

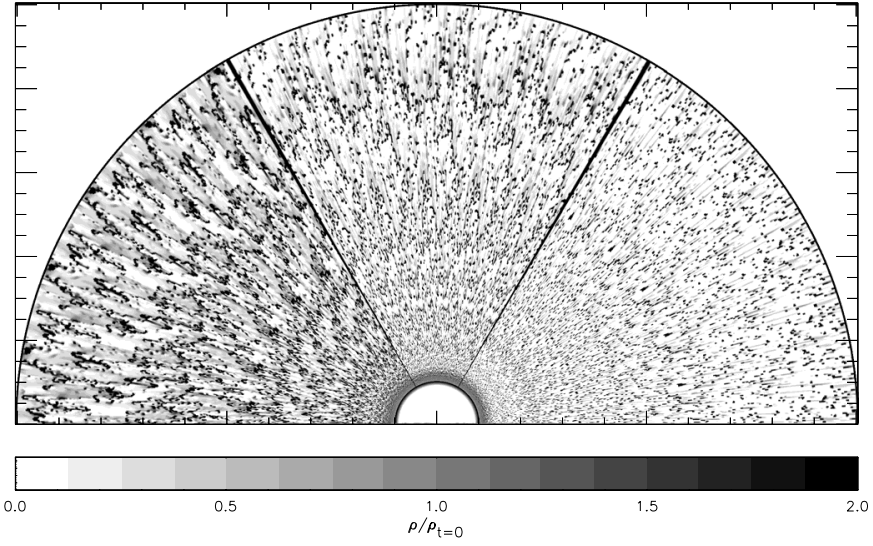

Fig. 1. Grayscale representation of the density contrast (normalized to the initial relaxed CAK model obtained with identical wind and stellar parameters) for a sequence of models with distinct viscosity amplitude $\alpha_{\text {vis }}$ (see Sect. 1 and Appendix), increasing clockwise over the range $0.01,0.1$ and 1 . For readability, each computed wedge is duplicated and stacked four times in azimuth, using the periodic boundary conditions employed in that direction.

More generally, this approach is also consistent with the notion that any moderate- to large-scale net asymmetry in the diffuse radiation field should be set by departures from the Sobolev limit defined by $v_{\text {th }} / v \rightarrow 0$. In particular, following the standard Sobolev approximation to next highest order in this small parameter leads to corrections that scale with $v_{\text {th }}$ times the gradient of the Sobolev optical depth $\tau \sim \kappa \rho v_{\text {th }} / v^{\prime}$, where $v^{\prime}$ is the velocity gradient along the direction of interest. For lateral components in which the gradient operator $\nabla \sim \partial / \partial \phi$, the combined derivatives lead to terms that scale as $v_{\text {th }} \partial^{2} v_{\phi} / \partial \phi^{2}$, as in Eq. (1).

Using this formulation, we have performed simulations analogous to the 2D-H+1D-R models described in DO-03, but now including, for negligible additional CPU costs, this viscous approximation (1) for the azimuthal diffuse radiation force, assuming various values of the dimensionless coefficient $\alpha_{\text {vis }}$. The radial and azimuthal grids are as defined in DO-03, with $n_{\phi}=60$ azimuthal zones of constant angular size $\delta \phi=0.2^{\circ}$, thus extending over a wedge of $\Delta \phi=n_{\phi} \times \delta \phi=12^{\circ}$, with periodic boundary conditions in azimuth.

As in the 2D-H+1D-R case, the simulations begin from a smooth, relaxed CAK model, from which there is initial formation of laterally coherent shell structures that arise from the strong radial instability of the line driving. Over time, these shells again break up from thin-shell and Rayleigh-Taylor instabilities, but with a final asymptotic form that is quite different depending on the assumed value of $\alpha_{\text {vis }}$. Figure 1 compares a representative snapshot of this asymptotic density structure (normalized by the density in the smooth CAK initial condition) for three models, divided into three display wedges of $60^{\circ}$ (representing 5 repetitions of the $12^{\circ}$ model computational wedges), corresponding in clockwise order to models with $\alpha_{\text {vis }}=0.01,0.1$, and 1. Comparison with the top panel of Fig. 1 in DO-03 shows that the structure in the low viscosity case $\alpha_{\text {vis }}=0.01$ (leftmost wedge of Fig. 1 here) is quite similar to that obtained in this previous model without any lateral 

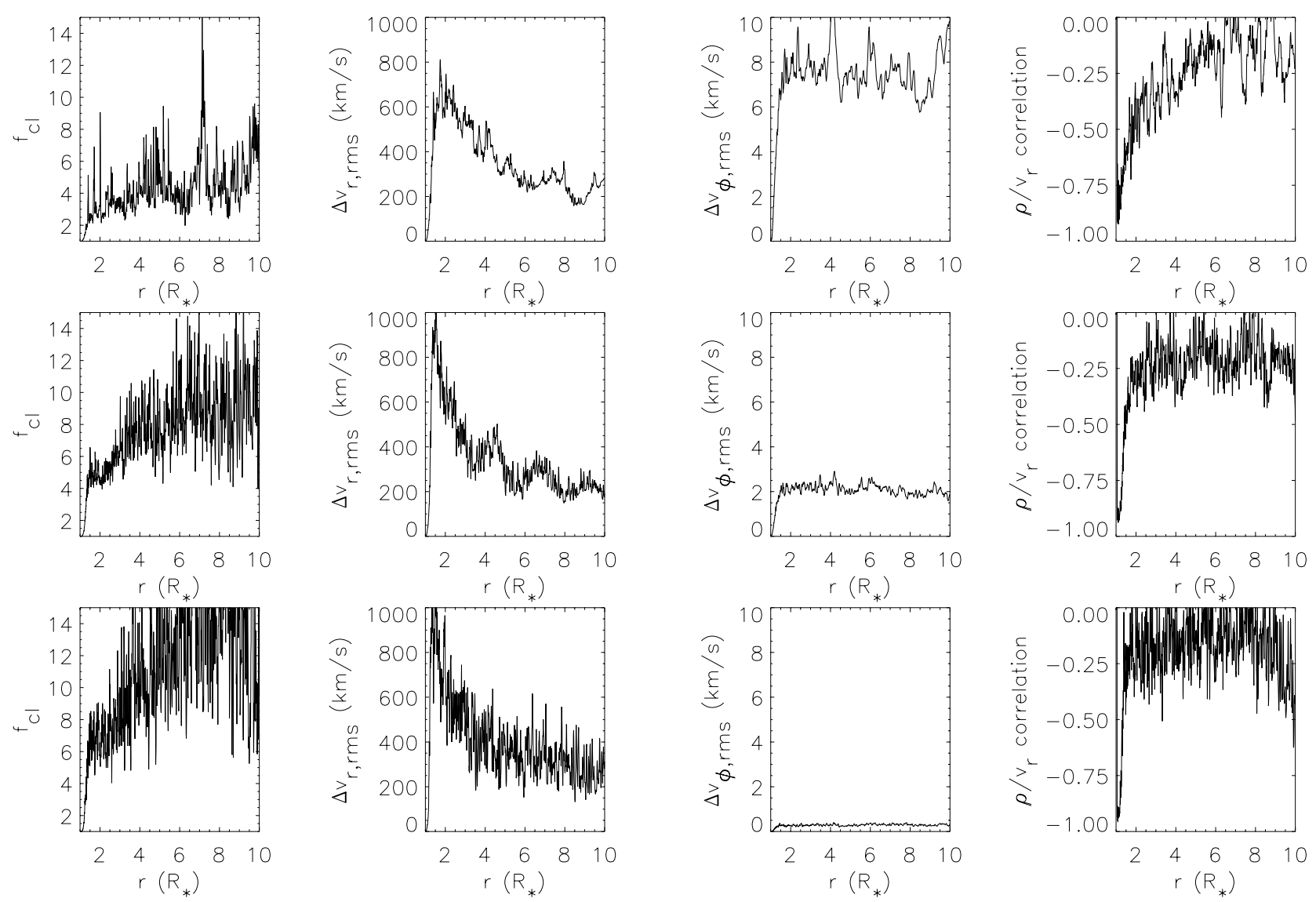

Fig. 2. Top row: radial variation of time- and angle-averages that characterize the nature of flow structure for the case with $\alpha_{\text {vis }}=0.01$. a) Clumping factor $f_{\mathrm{cl}}$; b) radial; and c) azimuthal velocity dispersion; and d) velocity-density correlation coefficient (Runacres \& Owocki 2002). Middle row: same as top for a model with $\alpha_{\text {vis }}=0.1$. Bottom row: same as top for a model with $\alpha_{\text {vis }}=1$. For ease of comparison, corresponding quantities are plotted over the same ordinate range.

radiation forces; but the more viscous cases with $\alpha_{\text {vis }}=0.1$ and $\alpha_{\text {vis }}=1$ (middle and right wedges of Fig. 1) are quite different, with progressively less radial elongation in filamentary structures.

To provide a more quantitative comparison, Fig. 2 shows representative statistical properties derived from time- and angle-averages of the density and velocity fields. For the case where $\alpha_{\text {vis }}=0.01$ (left wedge in Fig. 1 and top row in Fig. 2), the amplitude of azimuthal velocity variations is slightly reduced, but otherwise the clumping factor $f_{\mathrm{cl}}$, the radial velocity dispersion $\Delta v_{r, \text { rms }}$, and the velocity-density correlation function are all similar to those given in DO-03 with no lateral viscous term $\left(\alpha_{\text {vis }}=0\right)$.

By contrast, for the stronger viscosity cases $\alpha_{\mathrm{vis}}=0.1$ and $\alpha_{\text {vis }}=1$ (middle and lower panels of Fig. 2), we see that the azimuthal velocity is markedly reduced, with associated changes also in the other statistical parameters. The inhibition of lateral motion in effect tends to isolate further each of the azimuthal coordinates. In this situation, when a shock occurs, material from below ramming into the dense structure is prevented from circumventing it by the stronger radiative viscosity in the lateral direction. This behavior becomes even more pronounced in the highest viscosity case $\left(\alpha_{\text {vis }}=1\right)$, for which the dense structures resemble both radially and azimuthally confined clumps, i.e. dots in 2D. As shown in the bottom row of Fig. 2, the azimuthal velocity dispersion is then reduced by a factor of 20-30 compared to the first case. This corresponds to about a tenth of the sound speed, i.e. three orders of magnitude less than the wind flow speed. We see also that the clumping and radial velocity dispersion increase significantly with $\alpha_{\mathrm{vis}}$, converging for high $\alpha_{\text {vis }}$ to values similar to those found in purely 1D non-Sobolev simulations. Then, the lateral communication becomes so inhibited that each direction is essentially sheltered from its neighbors, and the 2D computed grid merely looks like a series of 1D non-Sobolev simulations stacked together in azimuth.

For a characteristic wind radius $r=6 R_{*}$, Fig. 3 compares the lateral variation of the azimuthal velocity and lateral radiation force for these three values of $\alpha_{\text {vis }}$. Note that the magnitude of the viscous forces are comparable (solid curves), even though the associated azimuthal velocity (dotted curves) is much smaller for the higher viscous coefficient. Note also that the sign of the viscous force depends on the concavity of the velocity variation.

A key result here is that the lateral diffuse radiation has a net effect that, in some sense, is opposite of what was anticipated, e.g., in DO-03. In particular, because the line-drag of diffuse radiation strongly damps lateral velocity variations on scales smaller than the lateral Sobolev length $l_{0} \approx r v_{\text {th }} / v$, DO-03 speculated that this could inhibit the operation of 

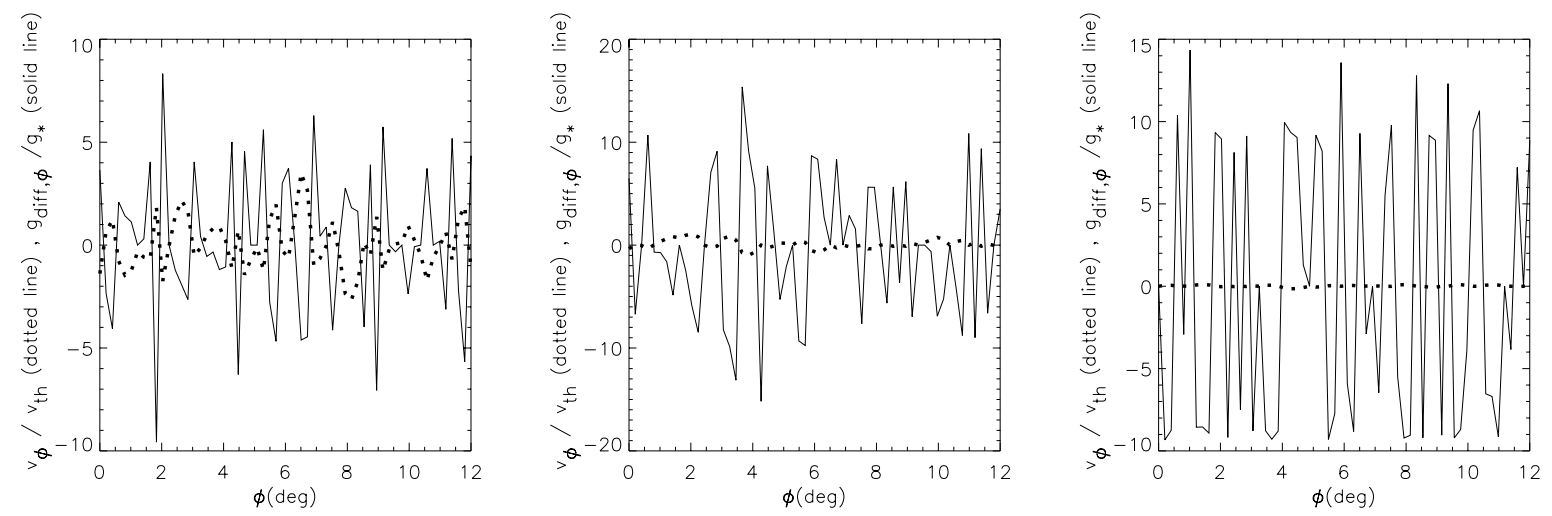

Fig. 3. Left: model with $\alpha_{\text {vis }}=0.01$. Azimuthal variation of the normalized viscous diffuse force $g_{\text {diff }, \phi}(r, \phi) / g_{*}(r)$, at $r=6 R_{*}$, and corresponding to the time-snapshot of Fig. 1. The lateral velocity, normalized to the thermal speed, is over-plotted (dotted line). Middle: same as left for a model with $\alpha_{\text {vis }}=0.1$. Right: same as left for a model with $\alpha_{\text {vis }}=1$.

Rayleigh-Taylor or thin-shell instabilities on this scale, and thus lead to a finite azimuthal coherence at the associated angle scale $\Delta \phi_{\text {Sob }} \approx l_{0} / r=v_{\text {th }} / v$. However, the above results show that such diffuse radiation damping, as modeled here in terms of a lateral viscosity, tends instead to limit further the lateral communication between neighboring azimuthal zones, and thus lead to an even greater level of lateral incoherence.

Since lateral line-drag does not limit structure to a finite azimuthal scale, we thus next consider how this might instead arise from the angle averaging of the backscattered radiation, which we next model in terms of an azimuthal averaging of the radial driving force.

\section{Azimuthal averaging of the radial radiation force}

A key limitation of the above lateral viscosity approach is that it still does not account for ways in which the lateral radiation transport might alter the radial component of the line-driving force. Indeed, since the radial driving at each azimuth is solved independently by strictly radial integrations of the line optical depth, there arises, through the diffuse component of the radial force, a backscattering feedback between the outer and inner wind that is effectively isolated and independent for each azimuthal zone. As such, both the previous $2 \mathrm{D}-\mathrm{H}+1 \mathrm{D}-\mathrm{R}$ and the present lateral viscosity models tend to develop structure that is independent for each azimuthal zone, implying a lateral incoherence down to the azimuthal grid scale.

More realistically, such radial backscattering should incorporate a nonzero level of lateral averaging, associated with transport along oblique rays that couple zones of different radius and azimuth. Unfortunately, as discussed in the Introduction, there are severe computational challenges to accounting for such oblique transport through direct integration along non-radial rays.

Thus, as a first approximate attempt to explore such effects, we have carried out here simulations in which the radial force is simply averaged over azimuth using a Gaussian filter with a tunable "smoothing angle" $\sigma_{\phi}$. At a given azimuthal
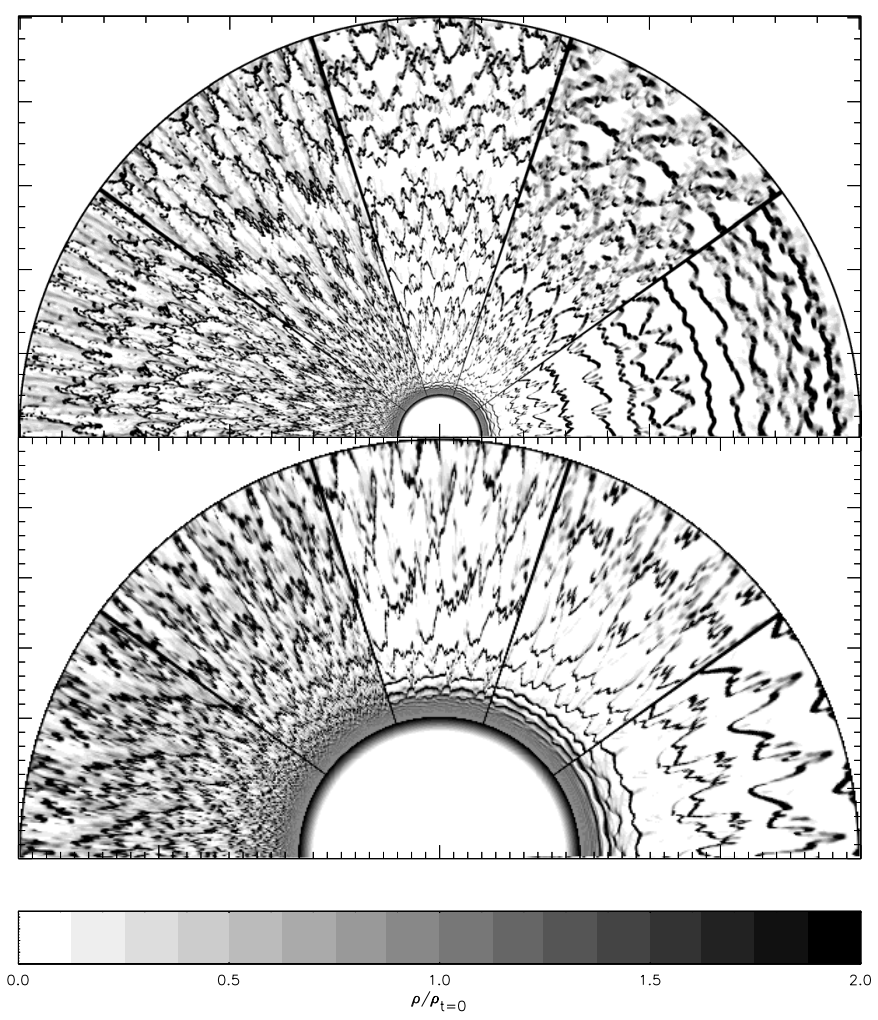

Fig. 4. Grayscale images of the density contrast normalized to an relaxed CAK model with identical wind and stellar parameters. At the top (bottom), models are shown only out to 10 (3) $R_{*}$. Each panel is composed of five different models (duplicated and stacked two times laterally to improve the visibility), ordered clockwise with increasing $\sigma_{\phi}$, i.e. $0,0.1,0.5,1$ and $1.5^{\circ}$.

coordinate $\phi_{j}$ and radial coordinate $r$, the smoothed radial force takes the form,

$g_{\mathrm{r}, \text { smooth }}\left(r, \phi_{j}\right)=\frac{\Sigma_{k} g_{\mathrm{r}}\left(r, \phi_{k}\right) \mathrm{e}^{-\left[\left(\phi_{j}-\phi_{k}\right) / \sigma_{\phi}\right]^{2}}}{\sum_{k} \mathrm{e}^{-\left[\left(\phi_{j}-\phi_{k}\right) / \sigma_{\phi}\right]^{2}}}$

Figure 4 compares the normalized density structure for simulations with $\sigma_{\phi}$ of $0,0.1,0.5,1$ and $1.5^{\circ}$, represented respectively by the clockwise sequence of wedges of width of $36^{\circ}$, each of which corresponding to 3 azimuthal periods of $\Delta \phi=12^{\circ}$ 
for each of the 5 model cases. The upper panels show results for the full simulated grid (out to $10 R_{*}$ ), while the lower panels zoom in on the region between the photosphere and $3 R_{*}$. Note that cases with a smoothing angle $\sigma_{\phi}$ less than the lateral grid resolution $\delta \phi=0.2^{\circ}$ show little departure from standard $2 \mathrm{D}-\mathrm{H}+1 \mathrm{D}-\mathrm{R}$ simulations. However, as $\sigma_{\phi}$ increases above $\delta \phi$, the coherence of wind structures becomes more pronounced. Indeed, for a smoothing angle of $\sigma_{\phi}=3^{\circ}$, or twice the maximum value shown in Fig. 4, we have found the lateral coherence extends across the full azimuthal wedge of $12^{\circ}$, so that the model effectively recovers the radially confined concentric shells corresponding to purely 1D (spherically-symmetric) instability simulations. Overall, this experimentation, although artificial, highlights the potential role of azimuthal averaging of radial driving in setting the lateral coherence of radiatively driven wind structures.

\section{Simulations using a 3-ray method for the nonlocal 2D line-force}

\subsection{Method formulation}

While the above models use a quite intricate Smooth-SourceFunction (SSF) escape-integral formulation for the radial force along each azimuth, their account of lateral transport effects on the radiative force is only phenomenological, through either a local viscosity or a simple azimuthal smoothing. A more consistent approach would carry out similar escape integrals along an appropriate set of oblique rays spanning the range from radial to lateral directions. Unfortunately, as already noted in Sect. 1, such a calculation presents severe computational challenges, stemming largely from the general misalignment of these rays with the nodes of the computational grid.

However, as first introduced by Owocki (1999), there is a specialized spatial grid that can allow a tractable, 3-ray, nonlocal formulation of both the radial and lateral components of the line-force. At any given grid point, the non-local escape probabilities are evaluated along one radial ray, plus two nonradial rays on opposite sides of the radial direction, set always to have a fixed impact parameter $p<R_{*}$ toward the stellar core. A key trick is to choose the radial spacing so that each nonradial ray intersecting a grid point with indices $\{i, j\}$ will also intersect other grid points $\{i \pm n, j \pm n\}$, for integer $n \geq 1$. This avoids the need to carry out a conceptually complex and computationally costly interpolation between a $(p, z)$ ray grid for the radiation transport, and the $(r, \phi)$ grid for the hydrodynamics. For uniform azimuthal spacing $\delta \phi$, such ray alignment occurs for radial grids satisfying

$$
r_{i}=\frac{p}{\cos \left[i \delta \phi+\arccos \left(p / R_{*}\right)\right.} .
$$

Figure 5 illustrates the grid and ray alignment for $\delta \phi=0.9^{\circ}$, $p / R_{*}=\sqrt{0.9}$ and $n_{r}=74$. Through spatial integration along the 3 such rays for each of the $n_{\phi}$ azimuthal zones, one obtains a "6-stream" description (i.e. in 2 directions along each of the 3 rays) for the required non-local escape probabilities from each of the $n_{r} \times n_{\phi}$ grid nodes.

Because each ray integration provides the escape probabilities along ca. $n_{r}$ grid nodes, the overall timing is now linear

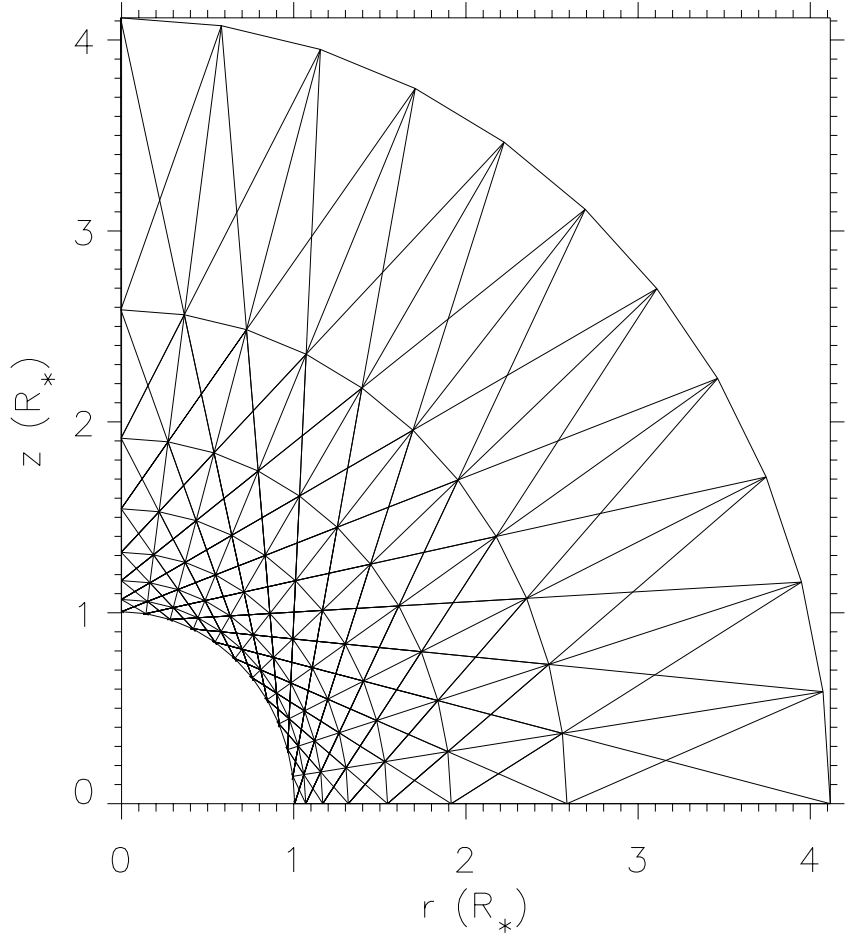

Fig. 5. Illustration of 3-rays with $p / R_{*}=0, \pm \sqrt{0.9}$ spaced azimuthally every $\delta \phi=0.9^{\circ}$, together with the radial grid spacing (circular arcs) that aligns the rays to intersect multiple $(r, \phi)$ grid nodes; this allows very efficient evaluation of the ray escape integrals needed for computation of the SSF line-force in 2D wind models. For clarity, only every ninth azimuthal or radial zone is shown.

(not quadratic, see Sect. 1) in $n_{r}$, scaling overall as $3 n_{\phi} n_{r} n_{x} \approx$ $10^{8}$ operations, or ca. a factor $10^{4}$ savings over the ca. $10^{12}$ operations estimated for a "brute-force", long characteristic computation in a non-aligned grid (cf. Sect. 1).

The difference in escape along the two nonradial rays provides a rough treatment of the lateral radiation transport. Because these rays are restricted to always impact within the stellar core radius $\left(p<R_{*}\right)$, they are best suited for approximating the direct component of the line-force; but in the crucial wind-acceleration region near the star, they have a substantial azimuthal component, and so also provide a rough approximation of the azimuthal part of the diffuse line-force, including, for example, the important lateral "line-drag" effect that is predicted to strongly damp small-scale azimuthal velocity variations (ROC). As the rays become increasingly radial at larger radii, this capacity to approximate the lateral, diffuse radiation is lost, but the 3 rays still provide a quite accurate representation of the finite-disk form for the direct line-force.

A more serious limitation arises from the severe loss of radial resolution at large radii, as demonstrated by the radial/ azimuthal grid aspect ratio,

$$
\frac{\delta r_{i}}{r_{i} \delta \phi} \approx \sqrt{\left(r_{i} / p\right)^{2}-1}
$$

which increases as $r_{i} / p$ at large radii. This means that smallscale radial structure can be relatively well resolved in the inner wind, but then becomes increasingly more damped by grid averaging in the outer wind. 
Table 1. Grid parameters used in the 3-ray SSF models. All simulations use 60 azimuthal zones and cover the first 10 stellar radii. We also give the extrema of the radial and azimuthal resolutions, to compare with the Sobolev length at mid wind-heights of ca. $0.01 R_{*}$ along the three rays.

\begin{tabular}{lccccc}
\hline \hline Model & \multicolumn{2}{c}{$\delta r / R_{*}$} & $n_{r}$ & $p / R_{*}$ & $\delta \phi(\mathrm{rad})$ \\
& $\min$. & $\max$. & & & \\
\hline $\mathrm{A}$ & $2.2 \times 10^{-4}$ & 0.074 & 1737 & $\sqrt{0.9}$ & $6.65 \times 10^{-4}$ \\
$\mathrm{~B}$ & $6.7 \times 10^{-4}$ & 0.101 & 1076 & $\sqrt{0.5}$ & $6.65 \times 10^{-4}$ \\
$\mathrm{C}$ & $4.5 \times 10^{-4}$ & 0.155 & 869 & $\sqrt{0.9}$ & $1.33 \times 10^{-3}$ \\
$\mathrm{D}$ & $1.3 \times 10^{-3}$ & 0.220 & 539 & $\sqrt{0.5}$ & $1.33 \times 10^{-3}$ \\
\hline
\end{tabular}

In our general scenario of self-excited instability, the nonlinear structure at larger radii plays a key role in seeding perturbations in the inner wind, through the backscattering feedback of the diffuse line-force. Thus it is important to minimize as much as possible this level of outer grid damping, by choosing as fine as possible radial grid, which in the 3-ray formalism here requires using as small as possible azimuthal grid size $\delta \phi$. In the simulations described below, we thus choose $\delta \phi \approx 10^{-3} \mathrm{rad} \approx 0.06^{\circ}$ (see Table 1 ) that are even finer than in previous models. For a characteristic wind Sobolev length $l_{0} \approx r v_{\text {th }} / v \approx 0.01 r$, this implies the radial grid size $\delta r$ is sufficient to resolve unstable structure near this scale out to a radius $r \approx p / 100 \delta \phi \approx 10 p \approx 7 R_{*}$.

\subsection{Linear perturbation test for 3-ray azimuthal diffuse force}

Before applying this 3-ray model in simulations of the nonlinear evolution of wind structure, it is instructive to examine its response to linear, test perturbations applied in a smooth, CAK initial background model. For this, let us compute the diffuse force for the simple case of a small amplitude lateral velocity perturbation $v_{\phi}$ that has a Gaussian variation in both radius and azimuth, centered on a grid coordinate $\left(r_{\mathrm{c}}, \phi_{\mathrm{c}}\right)$, with the corresponding widths $\Delta r_{\mathrm{c}}$ and $\Delta \phi_{\mathrm{c}}$,

$v_{\phi}(r, \phi)=v_{\text {th }} \exp \left[-\left(\frac{r-r_{\mathrm{c}}}{\Delta r_{c}}\right)^{2}-\left(\frac{\phi-\phi_{\mathrm{c}}}{\Delta \phi_{\mathrm{c}}}\right)^{2}\right]$.

Specifically, let us choose a representative radius $r_{\mathrm{c}}=1.5 R_{*}$, with $\phi_{\mathrm{c}}$ set at the mid-angle of the azimuthal grid, and then consider two cases distinguished by the relative scale of the assumed widths. For both cases, the 3-ray grid parameters $p$ and $\delta \phi$ are taken from the set B in Table 1 .

The first case assumes a very small scale for both the radial and azimuthal widths, $\Delta r_{c}=0.0005 R_{*}$ and $\Delta \phi_{0}=0.0001 \mathrm{rad}$, smaller in fact than the corresponding grid sizes, so that in effect the perturbation is essentially confined to the single grid point at $\left(r_{\mathrm{c}}, \phi_{\mathrm{c}}\right)$. Since both scales are thus also much smaller than the characteristic Sobolev length $l_{0} \approx r v_{\text {th }} / v \approx 0.01 r$, the response should follow the direct damping scaling given by Eq. (A.4) of the linear perturbation analysis in Appendix A. The lower panel of Fig. 6 shows just this type of scaling for this small-width case of the 3-ray numerical model, with the
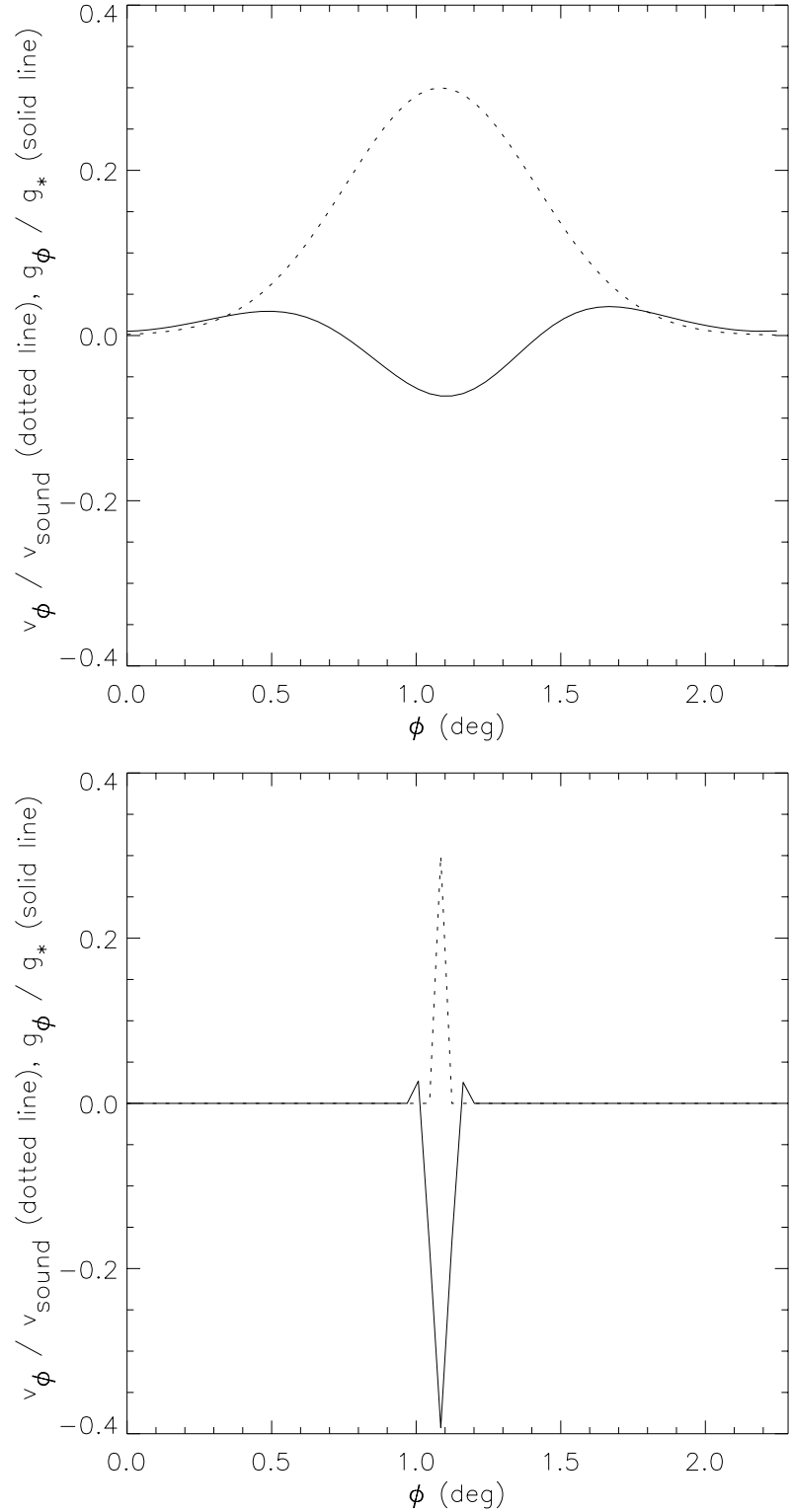

Fig. 6. Projected diffuse acceleration in the lateral direction from the two lateral rays (solid line) for a long- (top) and short- (bottom) wavelength velocity perturbation (dotted line). For both cases, we use a relaxed CAK radial velocity.

resulting azimuthal force (solid curve) varying with the negative of the azimuthal velocity perturbation (dashed curve).

The second case assumes a very large radial extent $\Delta r_{\mathrm{c}}=0.2 R_{*}$, and an azimuthal extent $\Delta \phi_{\mathrm{c}}=0.008 \mathrm{rad}$ that corresponds roughly with the characteristic Sobolev angle $l_{0} / r \approx 0.01$. With such comparatively large scales, the linear perturbation analysis in Appendix A now predicts the response to have the diffusive scaling (Eq. (A.5)), which implies a force that varies as the second derivative of the applied Gaussian velocity perturbation. The upper panel of Fig. 6 shows that the 3 -ray response in this larger-width case does indeed have just this type of scaling.

Overall, these tests demonstrate that, despite the inherently restricted nature of the angle quadrature, the 3-ray method does properly reproduce the appropriate scalings for the azimuthal 
component of the diffuse line-force. We thus now proceed to apply this method in simulations of the nonlinear evolution of the $2 \mathrm{D}$ flow structure.

\subsection{3-ray nonlinear simulation results}

We have used this 3-ray method to carry out 2D simulations for the nonlinear evolution of wind structure for four different combinations of the two basic grid parameters, namely the azimuthal resolution $\delta \phi$ and the impact parameter $p$ of nonradial rays, with values summarized in Table 1 . Both choices of the azimuthal grid size, $\delta \phi=1.33 \times 10^{-3}$ and $6.65 \times 10^{-4} \mathrm{rad}$, are fine enough to well resolve the characteristic lateral Sobolev angle, $l_{0} / r \approx 0.01$. The fine angle resolution is chosen in part because in this 3-ray method, a smaller $\delta \phi$ implies, for any given $p$, a finer radial resolution $\delta r$ (Eq. (4)). A larger $p$ also implies a finer radial grid (cf. Eq. (4)), as well as a more azimuthal orientation of the nonradial rays, especially near the stellar surface; this gives a greater sensitivity to lateral variations. The initial investigations by Owocki (1999) found the relaxed wind properties could depend quite strongly on the value of $p$. Our two choices of $p=\sqrt{0.5}$ and $p=\sqrt{0.9}$ allow us to further investigate this sensitivity.

In addition to the basic parameters, Table 1 also summarizes the number of grid points needed to reach the fixed outer radius $R_{\max }=10 R_{*}$, as well as the extrema of the radial resolution for each model. The latter reveal that the ray-projected Sobolev length is very well resolved at low heights, but unresolved near the outer grid radius. Apart from these differing grid properties, all four models have the same wind and stellar parameters as the models presented in DO-03, and in the previous two sections. To isolate physical effects that result solely from the use of a 3-ray radiation force, we also run all four models using just the radial ray transport; these thus represent 2D-H+1D-R models, but run on the (quite distinctive) 3-ray spatial grids.

Figure 7 shows a montage of grayscale images of the relaxed wind density for the four models, each normalized to the starting conditions obtained with an equivalent CAK model. The top row shows the full radial extent (to $R_{\max }=10 R_{*}$ ), while the bottom row zooms into the inner wind region (to $R_{\max }=3 R_{*}$ ). The left (right) column corresponds to 3-ray (1-ray) simulations. Each panel is divided into four wedges, corresponding respectively to models A, B, C and D stacked clockwise from the vertical, i.e. in order of decreasing radial resolution.

For the lower resolution models $(\mathrm{C}$ and $\mathrm{D})$, note that the shell structure is never really disrupted, irrespective of the impact parameter $p$, and, moreover, irrespective of the inclusion or neglect of lateral transport. In contrast, for the higher resolution models (A and B), the role of the diffuse force is quite apparent from a comparison of the left (3-ray) and right (1-ray) columns, especially if one focuses on the bottom-row panels that concentrate on the more well-resolved, inner wind. Most notably, in this region the lateral coherence is much greater when lateral transport is taken into account. However, there does not seem to be a significant dependence on the choice of impact parameter, with shell break-up starting at similar heights of ca. $2 R_{*}$ in both models $\mathrm{A}$ and $\mathrm{B}$.

We emphasize that, because of the degraded radial resolution and increasingly radial orientation of the oblique rays, the structure forms seen in the outer regions of these models are probably not very realistic. However, the inner regions have high resolution in both angle and radius, and thus we believe it is significant to find that the models with lateral transport can retain a lateral coherence that extends well beyond the azimuthal grid scale. This is quite distinct from the results of both 2D-H+1D-R models (cf. DO-03 Fig. 1 and the left and right columns of Fig. 7 here) and the models of Sect. 2, which use a viscous form for the lateral diffuse force. These results thus encourage further investigation of the effect of lateral transport in setting a finite lateral scale of instability-generated flow structure in line-driven stellar winds.

\section{Conclusions, summary, and future outlook}

This paper is a continuation of our ongoing efforts to develop multi-dimensional models for the nonlinear evolution of structure arising from the line-driven instability in hot-star winds. Building upon the previous $2 \mathrm{D}-\mathrm{H}+1 \mathrm{D}-\mathrm{R}$ simulations of DO-03, which carry out $2 \mathrm{D}$ hydrodynamics but use a $1 \mathrm{D}$, radial form for the line-transfer and force, we develop here approximate methods to account for two key effects expected in multi-dimensional treatments of the line-force, namely lateral line-drag and lateral averaging.

A summary of key results is as follows:

1. The lateral drag effect of diffuse line-radiation can be well modeled by casting the azimuthal line-force in a parallel viscosity form that scales with the second spatial derivative of the azimuthal velocity. For viscous coefficients of order the values derived from linear perturbation analysis, this does indeed lead to a strong damping of lateral velocity variations in $2 \mathrm{D}$, nonlinear simulations of the line-driven instability.

2. However, contrary to previous expectations, this reduction of lateral flow does not lead to a coherence at a characteristic Sobolev angle (ca. $1^{\circ}$ ) associated with the strongest damping. Instead, it tends to further isolate the azimuthal zones of the model, and so again leads to strong lateral incoherence down to the grid scale.

3. Lateral coherence may instead arise from the lateral averaging associated with the radial driving, as demonstrated here from heuristic models that explicitly impart an azimuthal smoothing to the radial driving force.

4. To account more consistently for both the lateral line-drag and lateral averaging effects, we have also experimented with a restricted, grid-aligned, 3-ray method that provides an approximate nonlocal treatment for both the radial and lateral components of the transport and line-force. Results for the highest resolution simulations indicate lateral averaging does tend to sustain an extended lateral scale in the resulting flow structure, but specifics of the results are left uncertain by the inherent limitations of the method. 

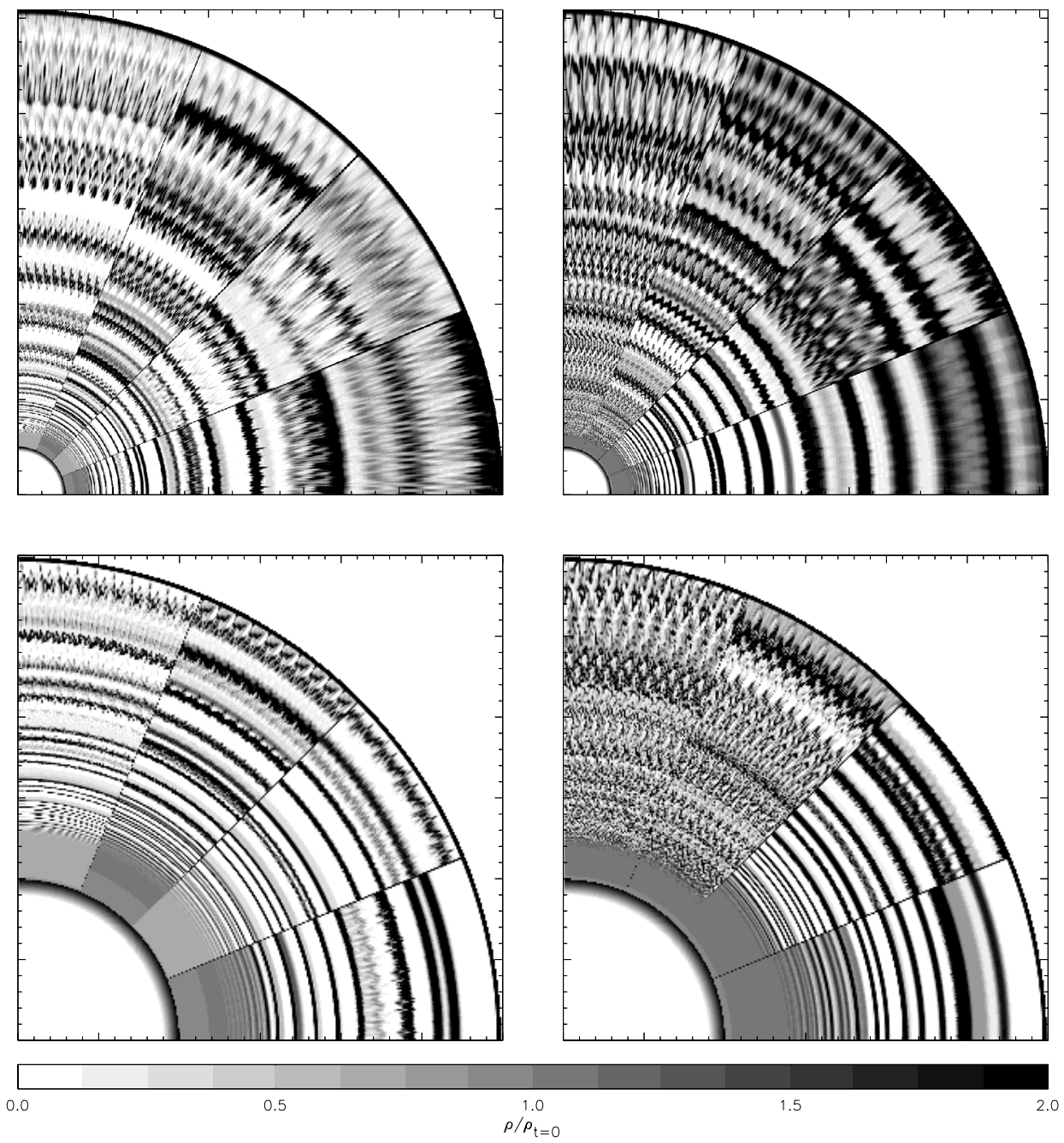

Fig. 7. Grayscale representation of the density contrast for relaxed non-Sobolev models computed with the 3-ray (left column) and 1-ray (right column) approaches. All models were run up until a total real time of $120000 \mathrm{~s}$. Panels in the top (bottom) row show models only out to 10 (3) $R_{*}$. Each panel is sub-divided into four wedges corresponding to models A, B, C, and D, stacked clockwise from the vertical in order of decreasing radial resolution (Table 1). As before, each computed angular wedge is duplicated a number of times so that the total angular extent of each wedge shown equals $22.5^{\circ}$, i.e. a quarter of the quadrant in each of the four panels. The key result shown here is the presence of laterally coherent wind structures close to the photosphere in the high resolution 3-ray models (A and B), while with the equivalent 1-ray model, one observes instead wind structures with no noticeable lateral coherence, i.e. of the order of the lateral numerical grid resolution.

Overall, a central conclusion is thus that lateral averaging of diffusion radiation appears promising as an effect that could set a distinct lateral scale to structures arising from the line-driven instability. However, further modeling with a less-restricted multi-ray method will be needed to confirm this idea, and to determine quantitatively the likely scale. In particular, as mentioned in the introduction and in DO-03, Rayleigh-Taylor or thin-shell instability likely plays a competing role in controlling such a lateral scale, although with a magnitude difficult to assess at present. We intend to explore development and application of such methods in our continuing work within this overall effort, with results to be reported in future papers in this series.

Acknowledgements. S.P.O. acknowledges support of NSF grant AST0097983, awarded to the University of Delaware.

\section{Appendix A: Linear analysis for lateral diffuse force}

Some key insights into the role of the multidimensional diffuse line force can be gleaned from the 3D linear perturbation analysis carried out by ROC. Let us begin with ROC's Eq. (A2) for the perturbed radiative acceleration tensor with components $T_{i j} \equiv \delta g_{i} / \delta v_{j}$, giving the $i$ 'th component of the radiative acceleration $\delta g_{i}$ arising from the $j$ 'th component of the velocity perturbation $\delta v_{j}$. For the diffuse component (stemming from the second term in the square bracket), we first note from symmetry and parity arguments that the tensor is purely real and diagonal, given by (for the pure scattering case $\epsilon=0$ )

$T_{i i}=-s \Omega\left\langle n_{i}^{2} \frac{\left(n_{i} k / 2 x_{*} Q_{0}\right)^{2}}{1+\left(n_{i} k / 2 x_{*} Q_{\mathrm{o}}\right)^{2}}\right\rangle$,

where the angle brackets denote averaging over solid angle, and we have assumed the usual case of longitudinal perturbations 
with $\boldsymbol{k} \| \delta \boldsymbol{v}$. The factor $Q_{0}$, defined in ROC Eq. (13), accounts for the angle variations of the velocity gradient in the smooth, spherically symmetric background flow, but for simplicity we have approximated this here by its value $Q_{0}=v_{o} /\left(r v_{\mathrm{th}}\right)=1 / l_{0}$ at the isotropic expansion point, where $\sigma \equiv d \ln v_{o} / d \ln r-1=$ 0 . This makes the order unity factor $s=\langle D\rangle /\langle D \mu\rangle=2 /(1+$ $\left.\mu_{*}\right)$, where $\mu_{*} \equiv \sqrt{1-R_{*}^{2} / r^{2}}$. The overall factor $-s \Omega$ gives a net damping rate that scales with the radial instability growth rate, $\Omega \equiv 2 x_{*} \alpha g_{o} / v_{\text {th }}$, where $\alpha \approx 0.6$ is the CAK exponent. For simplicity we henceforth take the blue-edge frequency $x_{*}$ to be unity.

In this work, we are interested in the lateral components $T_{11}=T_{22}$, representing variations along the azimuthal direction, for which the direction cosine in standard spherical coordinates $(\theta, \phi)$ takes the form $n_{1} \equiv n_{\phi}=\sin \theta \cos \phi$. Then defining $K \equiv k / 2 Q_{0}$, the integrals required for evaluation of the angle averaging take the form,

$\frac{\delta g_{\phi}}{\delta v_{\phi}}=-\frac{s \Omega}{4 \pi} \int_{0}^{2 \pi} \mathrm{d} \phi \int_{-1}^{1} \mathrm{~d} \mu \frac{K^{2} \cos ^{4} \phi\left(1-\mu^{2}\right)^{2}}{1+K^{2} \cos ^{2} \phi\left(1-\mu^{2}\right)}$,

where $\mu \equiv \cos \theta$. From numerical evaluation of these integrals, we find that this can be approximated (within ca. 10\%) by the simple form,

$\frac{\delta g_{\phi}}{\delta v_{\phi}} \approx-\frac{s \Omega}{3} \frac{K^{2}}{1+K^{2}}=-\frac{s \Omega}{3} \frac{k^{2}}{4 Q_{0}^{2}+k^{2}}$.

In the short-wavelength limit $k \gg 2 Q_{0}$, this gives

$\frac{\delta g_{\phi}}{\delta v_{\phi}} \approx-\Omega_{\text {damp }} \equiv-\frac{s \Omega}{3}=-\frac{2 \alpha s g_{o}}{3 v_{\text {th }}} ; k \gg 2 Q_{0}$,

where $\Omega_{\text {damp }}$ represents a damping rate associated with the lateral line-drag of this diffuse radiation.

In the long-wavelength limit $k \ll 2 Q_{0}$, we find

$\frac{\delta g_{\phi}}{\delta v_{\phi}} \approx-\frac{\Omega_{\mathrm{damp}} k^{2}}{4 Q_{0}^{2}} \approx-(\alpha s / 6)\left(v_{\mathrm{th}} r\right) k^{2} ; k \ll 2 Q_{0}$,

where the latter equality uses the approximation $g_{o} \approx v_{o}^{2} / r$. Note that this is just the form that would arise from a parallel viscosity with kinematic viscosity coefficient proportional to $v_{\mathrm{th}} r$, as assumed in Eq. (1). This thus forms a key motivation for the lateral viscosity approach used in Sect. 2 to model the 2D nonlinear development of structure arising from the strong radial instability of line driving.

The limiting forms (A.4) and (A.5) of Eq. (A.3) also provide a means to understand the small- and large-scale linear perturbation tests done for the 3-ray SSF method in Sect. 4.2. For the case in which the Gaussian perturbation width $r \Delta \phi_{o}=0.0005 r$ is much smaller than the lateral Sobolev length $l_{0}=r v_{\text {th }} / v_{o} \approx 0.01 r$, the lower panel of Fig. 6 shows that $g_{\phi} \approx-0.5 g_{*} v_{\phi} / v_{\mathrm{th}}$, which for a characteristic CAK acceleration $g_{o} \approx g_{*} /(1-\alpha)$ is quite consistent with the scaling given in Eq. (A.4) for this small-scale limit.
However, for the larger scale perturbation, the upper panel of Fig. 6 shows that the force response scales as the second derivative of the original Gaussian velocity perturbation, consistent with the viscous scaling predicted in the large-scale limit of Eq. (A.5).

\section{References}

Castor, J. I., Abbott, D. C., \& Klein, R. I. 1975, ApJ, 195, 157 (CAK) Chlebowski, T. 1989, ApJ, 342, 1091

Dessart, L., \& Owocki, S. P. 2002a, A\&A, 383, 1113

Dessart, L., \& Owocki, S. P. 2002b, A\&A, 393, 991

Dessart, L., \& Owocki, S. P. 2003, A\&A, 406, 1 (DO-03)

Dessart, L. 2004a, in Evolution of Massive Stars, Mass Loss and Winds, ed. M. Hedari-Malayeri, P. Stee, \& J.-P. Zahn, EAS Science Ser., 13, 251

Dessart, L. 2004b, A\&A, 423, 693

Eversberg, T., Lépine, S., \& Moffat, A. F. J. 1998, ApJ, 494, 799

Feldmeier, A. 1995, A\&A, 299, 523

Feldmeier, A., Puls, J., \& Pauldrach, A. 1997, A\&A, 322, 878

Feldmeier, A., \& Shlosman, I. 1999, ApJ, 526, 344

Gayley, K. G. 1995, ApJ, 454, 410

Gayley, K. G., Owocki, S. P., \& Cranmer, S. R. 1995, ApJ, 442, 296

Gayley, K. G., Owocki, S. P., \& Cranmer, S. R. 1997, ApJ, 475, 786

Gomez, E., \& Williams, R. 2003, MNRAS 344, 725

Grady, C. A., Bjorkman, K. S., \& Snow, T. P. 1987, ApJ, 320, 376

Howarth, I. D., \& Prinja, R. K. 1989, ApJS, 69, 527

Lépine, S., \& Moffat, A. F. J. 1999, ApJ, 514, 909

Lucy, L. B., \& Solomon, P. 1970, ApJ, 159, 879

Lucy, L. B. 1982, ApJ, 255, 278

Lucy, L. B. 1984, ApJ, 274, 372

Lucy, L. B., \& White, R. 1980, ApJ, 241, 300

Mihalas, D. 1978, Stellar Atmospheres, Second Edition (San Francisco: Freeman)

Owocki, S. P., \& Rybicki, G. B. 1984, ApJ, 284, 337

Owocki, S. P., \& Rybicki, G. B. 1985, ApJ, 299, 265

Owocki, S. P., Castor, J. I., \& Rybicki, G. B. 1988, ApJ, 335,914

Owocki, S. P. 1991, in Stellar Atmospheres: Beyond Classical Models, 235

Owocki, S. P., \& Puls, J. 1996, ApJ, 462, 894

Owocki, S. P., \& Puls, J. 1999, ApJ, 510, 355

Owocki, S. P., Cranmer, S. R., \& Gayley, K. G. 1996, ApJ, 472, 115

Owocki, S. P. 1999, in Variable and Non-spherical Stellar Winds in Luminous Hot Stars (Springer), Lecture Notes Phys., 523, 29

Petrenz, P., \& Puls, J. 2000, A\&A, 358, 956

Proga, D., Stone, J., \& Drew, J. 1999, MNRAS, 310, 476

Proga, D., \& Kallman, T. 2004, ApJ, 616, 688

Robert, C. 1992, Ph.D. Thesis, Univ. Montréal

Rybicki, G. B., Owocki, S. P., \& Castor, J. I. 1990, ApJ, 349, 274 (ROC)

Runacres, M. C., \& Owocki, S. P. 2002, A\&A, 381, 1015

Smith, N., Davidson, K., Gull, T. R., Ishibashi, K., \& Hillier, D. J. 2003, ApJ, 586, 432

Stone, J. M., \& Norman, M. J. 1992, 80, 753

van der Hucht, K., \& Williams, P. 1995, Wolf-Rayet Stars: Binaries, Colliding Winds, Evolution (Dordrecht: Kluwer), IAU Symp., 163 van Noort, M., Hubeny, I., \& Lanz, T. 2002, ApJ, 568, 1066 Woodward, P. R., \& Colella, P. 1984, J. Comput. Phys., 54, 115 